\title{
Questioning the 'neuroprotective' hypothesis: does drug treatment prevent brain damage in early psychosis or schizophrenia?
}

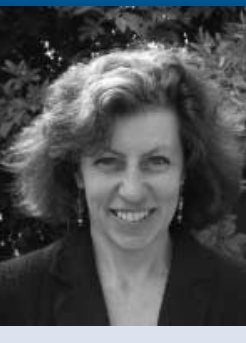

\author{
Joanna Moncrieff
}

\section{Summary}

The idea that psychotic disorders are characterised by progressive neurodegeneration that can be reversed by drug treatment is used to justify early treatment of increasing numbers of mostly young people. I argue that there is little evidence to support the view that old- or new-generation antipsychotics are 'neuroprotective', and some evidence that the drugs themselves may be responsible for the decline in brain matter observed in some studies.

\section{Declaration of interest}

J.M. is the co-chair person of the Critical Psychiatry Network
Joanna Moncrieff (pictured) is a senior lecturer at University College London and a consultant psychiatrist at the North East London Foundation Trust.

Almost 7 million prescriptions for antipsychotic drugs were issued outside hospital in England in 2008, an increase of 48\% since 1998. ${ }^{1}$ Data from the USA also indicate dramatically increased rates of prescription of antipsychotics to young people over recent years. ${ }^{2}$ Ideas about the benefits of early drug treatment in psychosis, along with other factors such as the marketing of bipolar disorder, ${ }^{3}$ are likely to have contributed to the increasing prescription of these drugs.

The importance of starting drug treatment early, and of continuing treatment in people who would like to stop, is often justified by the prevalent belief that schizophrenia or psychosis involves a progressive loss of brain tissue that can be arrested or reduced by treatment with antipsychotic drugs. ${ }^{4}$ Accordingly, the drugs are sometimes referred to as having 'neuroprotecive' properties. This belief is founded on a variety of evidence, all of which is debatable and open to other interpretations.

\section{Brain imaging research}

The main area believed to support the 'neuroprotection' hypothesis is brain imaging research illustrating that people with schizophrenia or psychosis show a progressive reduction in brain volume and an enlargement of ventricles and cerebral spinal fluid. All such studies, however, involve people who have taken longterm antipsychotic medication. A large study showing greater decline in brain volume in patients with first-episode psychosis taking haloperidol compared with olanzapine has been interpreted as indicating the neuroprotective effects of olanzapine. ${ }^{5}$ The authors acknowledged, however, that the study might also be interpreted as demonstrating differential drug-induced changes. Supporting the latter interpretation, a study of macaque monkeys treated with therapeutic doses of olanzapine and haloperidol for 18 months found an $8 \%$ reduction in mean fresh brain weight in monkeys treated with haloperidol and $11 \%$ in those on olanzapine compared with non-drug-treated controls. ${ }^{6}$

The great majority of studies of individuals who are drugnaive or have received only minimal treatment, do not show the global deficit in brain volume commonly associated with patients who have received drug treatment, including, most importantly, the only three studies involving people with long-term conditions who had not been exposed to drug treatment. ${ }^{7}$ Results from two studies of people considered to be at high risk of psychosis are also believed to demonstrate progressive brain tissue loss prior to and in the early stages of psychotic illness. Many of the Australian cohort, however, were taking antipsychotic medication during some of the follow-up period. ${ }^{8}$ In the Edinburgh study, high-risk individuals who progressed to psychosis showed greater temporal lobe reduction than those who did not progress, but did not differ from controls. ${ }^{9}$ Overall, therefore, imaging studies provide little evidence of progressive brain tissue loss in non-drug-treated patients with schizophrenia. Moreover, the effects of drug treatment have not been excluded as a cause of the tissue loss seen in some studies of drug-treated patients.

\section{Neuropathological findings}

Post-mortem studies have generally not found the large-scale neuronal loss and gliosis characteristic of neurodegenerative disorders in the brains of people with schizophrenia, although there is some evidence of local reduction of neuronal sub-populations and local loss of dendritic spines and length. ${ }^{10}$ Again, however, all studies have involved people on long-term medication. Some studies with drug-naive patients have found reduced serum levels of brain derived neurotrophic factor (BDNF), ${ }^{11}$ but this finding is not specific to schizophrenia, and BDNF has also been found to be involved in mood disorders and the stress response. Although schizophrenia has been suggested to involve increased or abnormal apoptosis, several studies confirm that markers of apoptosis in neurodegenerative disorders such as Alzheimer's disease are not raised in post-mortem tissue from patients with chronic schizophrenia, and the role of apoptosis earlier on in the condition remains speculative. ${ }^{12}$

\section{Duration of untreated psychosis}

Another frequently cited, indirect strand of evidence for the hypothesis that schizophrenia arises from progressive brain pathology that can be arrested by drug treatment is the association 
found in some studies (although not all) between the duration of untreated psychosis and outcome. However, it has long been recognised that conditions with a long and insidious early course are frequently more severe than those with a sudden onset. Duration of untreated psychosis is strongly related to mode of onset $^{13}$ and other characteristics of the psychosis itself, ${ }^{14}$ which makes it likely that it is simply a proxy measure of a more severe underlying condition. Research on duration of untreated psychosis and outcome has not adequately controlled for mode of onset and other potential confounding factors. ${ }^{15}$

Trials of early intervention have shown some positive results, but have not analysed the role of medication over and above other aspects of the intervention, and long-term results are disappointing (reviewed in Bosanac et al). ${ }^{15}$ Two trials of drug treatment for young people at high risk of psychosis suggested that the rate of conversion to psychosis was reduced, although not by a statistically significant degree in the larger of the two trials. ${ }^{16} \mathrm{~A}$ larger naturalistic study has so far found that drug treatment has not reduced the onset of psychosis. ${ }^{17}$

\section{Evidence on neuroprotection}

At a neuropathological level, ideas about drug treatment being neuroprotective developed because of evidence that some psychiatric drugs, notably lithium, increase activity in some pathways associated with neurotrophism and neurogenesis. ${ }^{18}$ The functional implications of these findings are unclear, however. One study, for example, found that although lithium reduced levels of kainate-induced excitotoxic motor neuron cell death, surviving cells were not undamaged. ${ }^{19}$ In any case, evidence of antipsychotics has not consistently demonstrated these sorts of effects. ${ }^{18}$ The view that new atypical antipsychotics have a particular neuroprotective effect may derive from an animal study of olanzapine and clozapine that detected upregulation of the neuroprotective Bcl-2 (B-cell lymphoma 2) protein. ${ }^{20}$ However, $\mathrm{Bcl}-2$ is also upregulated in neurodegenerative disorders such as Alzheimer's disease, where it is regarded as evidence of a compensatory mechanism. Other studies have not replicated this finding, but one study found increased activity of the pro-apoptotic protease capase- 3 in animals treated with old and new antipsychotics. ${ }^{21}$

Effects of antipsychotics, particularly atypicals, on cognitive function is also cited as evidence of their neuroprotective effects, but whether or not any antipsychotics improve cognition independently of symptoms remains uncertain. The suggested superior effects of atypical antipsychotics have also been questioned by the findings of the Clinical Antipsychotic Trials of Intervention Effectiveness (CATIE) study, in which the greatest long-term improvements in cognitive function were seen in the perphenazine group. ${ }^{22}$

In contrast to ideas about neuroprotective effects, there is suggestive evidence that antipsychotics reduce brain tissue volume, as described above, ${ }^{7}$ and they are known to cause the pathological brain syndrome known as tardive dyskinesia. Research suggests that as well as involuntary movements, tardive dyskinesia is associated with general cognitive decline, suggesting that antipsychotics can induce generalised brain dysfunction. ${ }^{23}$

\section{Conclusions}

Antipsychotics are undoubtedly useful in suppressing the symptoms of psychotic conditions, but the evidence presented here suggests there is little basis to the belief that they reverse an underlying neurodegenerative process in people with schizophrenia or psychosis. Neuropathological studies do not support the idea that antipsychotic drugs, including the new or atypical antipsychotics, have neuroprotective effects. Some research suggests they may even contribute to the decline in brain volume seen in people with these diagnoses, and they are known to induce neurological damage in the form of tardive dyskinesia in some long-term users. The idea that antipsychotic drugs are neuroprotective should not therefore be used as a justification for prescribing or continuing to prescribe them, if other considerations do not also support their use. Psychiatrists should be particularly cautious about the use of antipsychotics in the early or prodromal stages of psychosis, where, as others have pointed out, there is the potential to do much harm. ${ }^{15}$

Joanna Moncrieff, MRBS, MSC, MD, MRCPsych, Department of Mental Health Sciences, University College London, 67-73 Riding House Street, London W1W 7EJ, UK. Email: j.moncrieff@ucl.ac.uk

First received 11 Aug 2010, accepted 15 Sep 2010

\section{References}

1 Information Centre for Health and Social Care. Prescription Cost Analysis 2008. NHS Information Centre, 2009.

2 Olfson M, Blanco C, Liu L, Moreno C, Laje G. National trends in the outpatient treatment of children and adolescents with antipsychotic drugs. Arch Gen Psychiatry 2006; 63: 679-85.

3 Healy D. The latest mania: selling bipolar disorder. PLOS Med 2006; 3: e185.

4 Lieberman JA. Is schizophrenia a neurodegenerative disorder? A clinical and neurobiological perspective. Biol Psychiatry 1999; 46: 729-39.

5 Lieberman JA, Tollefson GD, Charles C, Zipursky R, Sharma T, Kahn RS, et al. Antipsychotic drug effects on brain morphology in first-episode psychosis. Arch Gen Psychiatry 2005; 62: 361-70.

6 Dorph-Petersen KA, Pierri JN, Perel JM, Sun Z, Sampson AR, Lewis DA. The influence of chronic exposure to antipsychotic medications on brain size before and after tissue fixation: a comparison of haloperidol and olanzapine in macaque monkeys. Neuropsychopharmacology 2005; 30: 1649-61.

7 Moncrieff J, Leo J. A systematic review of the effects of antipsychotic drugs on brain volume. Psychol Med 2010; 40: 1409-22.

8 Takahashi T, Wood SJ, Yung AR, Soulsby B, McGorry PD, Suzuki M, et al. Progressive gray matter reduction of the superior temporal gyrus during transition to psychosis. Arch Gen Psychiatry 2009; 66: 366-76.

9 Lawrie SM, Whalley HC, Abukmeil SS, Kestelman JN, Miller P, Best JJK, et al. Temporal lobe volume changes in people at high risk of schizophrenia with psychotic symptoms. Br J Psychiatry 2002; 181: 138-43.

10 Jarskog LF, Miyamoto S, Lieberman JA. Schizophrenia: new pathological insights and therapies. Annu Rev Med 2007; 58: 49-61.

11 Chen DC, Wang J, Wang B, Yang SC, Zhang CX, Zheng YL, et al. Decreased levels of serum brain-derived neurotrophic factor in drug-naive first-episode schizophrenia: relationship to clinical phenotypes. Psychopharmacology (Berl) 2009; 207: 375-80

12 Jarskog LF, Glantz LA, Gilmore JH, Lieberman JA. Apoptotic mechanisms in the pathophysiology of schizophrenia. Prog Neuropsychopharmacol Biol Psychiatry 2005; 29: 846-58.

13 Morgan C, Abdul-Al R, Lappin JM, Jones P, Fearon P, Leese M, et al. Clinical and social determinants of duration of untreated psychosis in the $\mathbb{E}$ SOP firstepisode psychosis study. Br J Psychiatry 2006; 189: 446-52.

14 Owens DC, Johnstone EC, Miller P, Macmillan JF, Crow TJ. Duration of untreated illness and outcome in schizophrenia: test of predictions in relation to relapse risk. Br J Psychiatry 2010; 196: 296-301.

15 Bosanac $P$, Patton GC, Castle DJ. Early intervention in psychotic disorders: faith before facts? Psychol Med 2010; 40: 353-8.

16 McGlashan TH, Zipursky RB, Perkins D, Addington J, Miller T, Woods SW, et al. Randomized, double-blind trial of olanzapine versus placebo in patients prodromally symptomatic for psychosis. Am J Psychiatry 2006; 163: 790-9.

17 Walker EF, Cornblatt BA, Addington J, Cadenhead KS, Cannon TD, McGlashan TH, et al. The relation of antipsychotic and antidepressant medication with baseline symptoms and symptom progression: a naturalistic study of the North American Prodrome Longitudinal Sample. Schizophr Res 2009; 115: 50-7. 
18 Hunsberger J, Austin DR, Henter ID, Chen G. The neurotrophic and neuroprotective effects of psychotropic agents. Dialogues Clin Neurosci 2009; 11: 333-48.

19 Caldero J, Brunet N, Tarabal O, Piedrafita L, Hereu M, Ayala V, et al. Lithium prevents excitotoxic cell death of motoneurons in organotypic slice cultures of spinal cord. Neuroscience 2010; 165: 1353-69.

20 Bai O, Zhang H, Li XM. Antipsychotic drugs clozapine and olanzapine upregulate $\mathrm{bcl}-2$ mRNA and protein in rat frontal cortex and hippocampus. Brain Res 2004; 1010: 81-6.
21 Jarskog LF, Glantz LA, Gilmore JH, Lieberman JA. Apoptotic mechanisms in the pathophysiology of schizophrenia. Prog Neuropsychopharmacol Biol Psychiatry 2005; 29: 846-58.

22 Keefe RS, Bilder RM, Davis SM, Harvey PD, Palmer BW, Gold JM, et al. Neurocognitive effects of antipsychotic medications in patients with chronic schizophrenia in the CATIE Trial. Arch Gen Psychiatry 2007; 64: 633-47.

23 Waddington JL, O'Callaghan E, Larkin C, Kinsella A. Cognitive dysfunction in schizophrenia: organic vulnerability factor or state marker for tardive dyskinesia? Brain Cogn 1993; 23: 56-70.

\section{Shabbir Amanullah}

The days of the conventional physician seem numbered and one can almost see the day when surgical operations will be performed by robots possibly monitored by a surgeon initially. Surgical emergencies will be dealt with by mobile surgical units that are self-contained and whiz around town performing operations quietly and efficiently. After all, most of the time diagnoses are based on symptom clusters and a well-programmed robot can do the same, with no room for errors due to anxiety, substance misuse, anger, exhaustion, etc., effectively using advanced imaging techniques. Of course, reporting will be outsourced!

Imagine an accident and emergency department with no emotional tension. No anger at the return of a difficult patient. Just a series of television screens and plug-in memory cards in the mobile units. Whole body scans for those with flu-like symptoms and no fears of cross-infections or iatrogenic infections. No more MRSA or VRA (unless we insist that they wear ties!).

It seems increasingly likely that with rapid progress in science we as physicians may be a dying breed. All except, possibly, psychiatry. Grief will be a major issue and so too robot phobias. Those of our medical colleagues looking for work may come for reminiscence therapy - 'Remember the good old days, when we used to ...' 'There will of course be those who grieve the loss of the human physician. My message to medical students: join psychiatry and remain employed! 